\title{
Erratum
}

\section{Learning probability distributions in continuous evolutionary algorithms - a comparative review}

STEFAN KERN, SIBYLLE D. MÜLLER, NIKOLAUS HANSEN, DIRK BÜCHE, JIRI OCENASEK and PETROS KOUMOUTSAKOS

Natural Computing 3: 77-112, 2004.

Some equations were printed incorrectly, which the publisher deeply regrets. Please note the corrected ones below.

Page 82. Equation (7) should read:

$$
c=10 /(n+20), \quad d=\max \left(1, \frac{3 \mu}{n+10}\right)+\mathrm{c}
$$

Page 83. Equation (14) should read:

$$
c_{c}=\frac{4}{n+4}, \quad c_{\sigma}=\frac{10}{n+20}, \quad d=\max \left(1, \frac{3 \mu}{n+10}\right)+\mathrm{c}_{\sigma}
$$

Page 90. Table 2 should read:

\begin{tabular}{|c|c|c|c|}
\hline & $N_{\text {parent }}$ & $N_{\text {offspr }}$ & other \\
\hline$(1+1)-\mathrm{ES}$ & 1 & 1 & $\alpha=2^{1 / n}$, success rate $1 / 5$ \\
\hline CSA-ES & $\mu=N_{\text {base }} / 2$ & $\lambda=N_{\text {base }}$ & $c=\frac{10}{n+20}, d=\max \left(1, \frac{3 \mu}{n+10}\right)+\mathrm{c}$ \\
\hline \multirow[t]{4}{*}{ CMA-ES } & \multirow[t]{4}{*}{$\mu=N_{\text {base }} / 2$} & \multirow[t]{4}{*}{$\lambda=N_{\text {base }}$} & $c_{\sigma}=\frac{10}{n+20}, d=\max \left(1, \frac{3 \mu}{n+10}\right)+\mathrm{c}_{\sigma}$ \\
\hline & & & 1 \\
\hline & & & $c_{c}=\overline{n+4}, c_{\mathrm{cov}}=\bar{\mu} \overline{(n+\sqrt{2})^{2}}$ \\
\hline & & & $+\left(1-\frac{1}{-}\right) \min \left(1, \frac{2 \mu-1}{2}\right)$ \\
\hline \multirow[t]{3}{*}{ IDEA } & $\tau N_{\text {base }}$ & $(1-\tau) N_{\text {base }}$ & $\tau=0.3, \lambda_{c}=0.5, \kappa=n-1$ \\
\hline & & & number of clusters \\
\hline & & & threshold for adding a cluster \\
\hline MBOA & $\tau N_{\text {base }}$ & $N_{\text {base }} / 2$ & $\tau=0.5$, window size $5 \%$ \\
\hline
\end{tabular}

Table 2. Strategy parameters of the algorithms 
Page 92. Table 3 should read:

Table 3. Test functions and initialization intervals (coordinate-wise), where $\boldsymbol{y}:=\left[\boldsymbol{o}_{1} \ldots\right.$, $\left.\boldsymbol{o}_{n}\right]^{T} \boldsymbol{x}$, i.e., $y_{i}=\boldsymbol{o}_{i}^{T} \boldsymbol{x}$, see text

\begin{tabular}{lll} 
Name & Function & Init \\
\hline Plane & $f_{\text {plane }}(\boldsymbol{x})=x_{1}$ & {$[0.5,1.5]^{n}$} \\
Diagonal plane & $f_{\text {planediag }}(\boldsymbol{x})=\frac{1}{n} \sum_{i=1}^{n} x_{i}$ & {$[0.5,1.5]^{n}$} \\
Sphere & $f_{\text {sphere }}(\boldsymbol{x})=\sum_{i=1}^{n} x_{i}^{2}$ & {$[-3,7]^{n}$} \\
Ellipsoid & $f_{\text {elli }}(\boldsymbol{x})=\sum_{i=1}^{n}\left(100^{\frac{i-1}{n-1}} y_{i}\right)^{2}$ & {$[-3,7]^{n}$} \\
Cigar & $f_{\text {cigar }}(\boldsymbol{x})=y_{i}^{2}+10^{4} \sum_{i=2}^{n} y_{i}^{2}$ & {$[-3,7]^{n}$} \\
Tablet & $f_{\text {tablet }}(\boldsymbol{x})=10^{4} y_{1}^{2}+\sum_{i=2}^{n} y_{i}^{2}$ & {$[-3,7]^{n}$} \\
Rosenbrock & $f_{\text {Rosen }}(\boldsymbol{x})=\sum_{i=1}^{n-1}\left(100 \cdot\left(x_{i}^{2}-x_{i+1}\right)^{2}+\left(x_{i}-1\right)^{2}\right)[-5,5]^{n}$ \\
Rastrigin & $f_{\text {Rastrigin }}(\boldsymbol{x})=10 n+\sum_{i=1}^{n}\left(y_{i}^{2}-10 \cos \left(2 \pi y_{i}\right)\right)$ & {$[-3,7]^{n}$} \\
Scaled Rastrigin & $f_{\text {Rastrigin10 }}(\boldsymbol{x})=10 n+\sum_{i=1}^{n}\left(\left(10^{\frac{i-1}{n-1}} y_{i}\right)^{2}\right.$ & {$[-3,7]^{n}$} \\
& \multicolumn{2}{c}{$\left.-10 \cos \left(2 \pi 10^{\frac{i-1}{n-1}} y_{i}\right)\right)$} \\
\hline
\end{tabular}

